\title{
Oncogenes in immune cells as potential therapeutic targets
}

This article was published in the following Dove Press journal: ImmunoTargets and Therapy

\author{
Gulnur K Zakiryanova' \\ Sarah Wheeler ${ }^{2}$ \\ Michael R Shurin ${ }^{2}$ \\ 'Department Biophysics and \\ Biomedicine, Faculty Biology and \\ Biotechnology, Al-Farabi Kazakh \\ National University, Almaty, \\ Kazakhstan; ${ }^{2}$ Division of Clinical \\ Immunopathology, Department of \\ Pathology, University of Pittsburgh \\ Medical Center, Pittsburgh, PA, USA
}

Correspondence: Gulnur K Zakiryanova; Department Biophysics and Biomedicine, Faculty Biology and Biotechnology, Al-Farabi Kazakh National University, Al-Farabi 7I, Bldg 6., Almaty 050040, Kazakhstan

Email gzakiryanova@gmail.com

Michael R Shurin

Division of Clinical Immunopathology, Department of Pathology, University of Pittsburgh Medical Center, Pittsburgh, PA, USA

Email shurinmr@upmc.edu

\begin{abstract}
The role of deregulated expression of oncogenes and tumor-suppressor genes in tumor development has been intensively investigated for decades. However, expression of oncogenes and their potential role in immune cell defects during carcinogenesis and tumor progression have not been thoroughly assessed. The defects in proto-oncogenes have been well documented and evaluated mostly in tumor cells, despite the fact that proto-oncogenes are expressed in all cells, including cells of the immune system. In this review, key studies from immune-mediated diseases that may be associated with oncogene signaling pathways are refocused to provide groundwork for beginning to understand the effects of oncogenes in and on the cancer-related immune system dysfunction.
\end{abstract}

Keywords: oncogenes, immune cells, NK cells, Myc, cancer

\section{Introduction}

Proto-oncogenes are normal genes involved in cell differentiation, growth, proliferation and apoptosis, and when mutated or overexpressed can cause normal cells to become cancerous. ${ }^{1}$ Tumor development and progression is associated with local and systemic immune suppression and dysregulation. Immune mechanisms play an integral role in the formation of tumor microenvironment, tumor growth and establishment of metastases. ${ }^{2}$ The main cause of immune abnormalities in cancer is often attributed to the suppressive factors produced by tumor cells or tumor-polarized immune and stromal cells. The role of oncogenes and onco-suppressors is well appreciated and studied in tumor development; however, their role in immune cell defects during carcinogenesis and tumor growth and progression is not well understood. Proto-oncogenes have been evaluated primarily in cancerous and premalignant cells, in spite of the fact that they are expressed in almost all cell types, including cells of the immune system. There are only a few reports focusing on the immune-modulating mechanisms of oncogenes expressed in cancer cells, immune cells or in the tumor microenvironment.

The carcinogenetic process is initiated by chemical, biological and/or physical carcinogens or spontaneous mutations and driven by the progressive accumulation of oncogenic mutations and epigenetic aberrations in the expression of many genes with various functions. Despite multiple mutations and epigenetic aberrations, the reversal of a small subset of these genes often significantly attenuates tumor growth. This is the basis of the "oncogene addiction" postulate ${ }^{3,4}$ that emphasizes the deceptive dependency of cancer on a small number of genes for the preservation of the malignant phenotype. ${ }^{5,6}$ Oncogene addiction has traditionally been thought of as a cell-intrinsic 
characteristic, such as proliferative arrest, apoptosis, differentiation and cellular senescence. However, recent data suggest that oncogene addiction may be dependent upon host immune-mediated mechanisms, including specific immune effector cells and cytokines regulating cancer cell senescence and tumor-associated angiogenesis. ${ }^{7}$ Preliminary work indicates that removal of a single oncogene may induce tumor cell death by a cell-intrinsic mechanism which is accompanied by liberating tumor-associated antigens, cytokines and immunomodulators that activate antigen-presenting cells and boost the antitumor immune responses. ${ }^{8}$ Targeted inactivation of the $M Y C, B C R-A B L$ or $H E R 2 /$ neu oncogenes correlates with tumor infiltration by CD4+ T cells, TGF- $\beta$ expression and inhibition of tumor growth..$^{9-11}$ Additionally, proto-oncogenes are expressed ubiquitously in many cell types, including immune cells, and their removal or inactivation can have a direct effect on the functions of immune cells in different pathophysiological conditions. For example, certain proto-oncogenes may be activated in systemic lupus erythematosus, Sjögren's syndrome, rheumatoid arthritis, progressive systemic sclerosis and dermatomyositis, supporting a hypothesis that oncogenes might play the role of "rheumogenes" at certain conditions. ${ }^{12}$ However, the activation of oncogenes in autoimmune conditions seems to be not associated with gene mutations, demonstrating that autoimmunity ${ }^{13,14}$ encompasses different mechanisms of oncogene activation than do many malignancies.

Understanding how molecules targeting oncogenes can harness context-dependent cellular functions is clinically important. Increasing our knowledge about oncogenedependent pathways in immune cells will provide direction for novel therapeutic application of existing and new agents in a variety of immune-mediated diseases, including cancer.

Another interesting aspect of "oncogenes and immunity" may be the role of oncogene-controllable immune responses in spontaneous cancer regression, which is a rare but welldocumented clinical phenomenon. Spontaneous regression is defined as the partial or complete disappearance of a tumor in the absence of any treatment capable of regression, and was reported in virtually all types of human cancers, although the greatest number of cases were reported in patients with neuroblastoma, renal cell carcinoma, malignant melanoma, basal cell carcinoma and other so-called immunogenic tumors. ${ }^{15,16}$ For instance, some estimations suggested that the rate of spontaneous regression in melanoma could be between $10 \%$ and $20 \%$, based on histological studies that showed that $25 \%$ of melanomas have evidence of partial regression, which suggests that melanomas can induce immune responses and that some patients display immunity to their melanomas that keep the tumor under some sort of control. ${ }^{17}$ While there is evidence of regression in as many as $50 \%$ of basal cell carcinomas of the skin, clinical studies on the effect of immunotherapy on basal cell carcinoma reported that tumors disappeared in $20 \%$ of patients on the placebo arm of the trial, indicating that about $20 \%$ of basal cell carcinomas undergo complete spontaneous regression. ${ }^{18}$

Various mechanisms are considered to be associated with this phenomenon, including suppression or loss of essential oncogene expression, ${ }^{19}$ tumor inhibition by growth factors or cytokines, induction of differentiation, inhibition of angiogenesis, hormonal mediation and tumor necrosis..$^{20,21}$ However, the mechanisms that are most often implicated in driving the spontaneous regression of cancer are believed to be associated with immune system activation or reactivation. ${ }^{21-24}$

However, one may suggest another mechanism, which has not been considered - activation of immune response, in particular innate immunity, associated with the ability of immune cells, including NK cells, to "sense" oncogeneic stress in the tumor microenvironment. In fact, the mechanisms that detect cellular stresses that are accompanied by tumorigenesis and that culminate in the recognition and, in some cases, the elimination of tumor cells, NK cells, and other lymphocytes that express NK cell receptors are well known. ${ }^{25}$ Thus, it is possible that lost oncogene expression or induced cancerous cell differentiation might provide a signal detected by NK cell receptor-expressing cells and that this signal is essential for initiating efficient tumor elimination. This pathway may also explain the absence of detectable $\mathrm{T}$ cell responses often described in cases of spontaneous cancer regression.

\section{The role of oncogenes in the regulation of immune cells: NK cells}

Analysis of immunological abnormalities in cancer is usually associated with investigations of immunosuppression induced by cancer cells and tumor stromal elements. The phenotypic and cytotoxic activity of NK cells in the tumor environment in vitro and in vivo has been repeatedly described ${ }^{26-28}$ with tumor-associated NK cells displaying marked phenotypic and functional alterations. Alterations include changed expression of NKp30, CD16, DNAM-1 and ILT2, decreased cytotoxicity and reduced IFN- $\gamma$ production. ${ }^{29-31}$ The presence of a $\mathrm{CD} 56^{\text {bright }} \mathrm{CD} 16^{+} \mathrm{NK}$ cell subset with unregulated expression of activating receptors, perforin molecules and high activity was also reported in the tumor-draining lymph nodes in melanoma patients. ${ }^{32}$ Similar alterations in peripheral blood NK cells from patients with solid tumors were also reported. ${ }^{33,34}$ 
In general, it is accepted that there is a direct correlation between the degree of immune alterations and the stage of disease. Recent data, however, suggest that there is no such correlation in a number of cases. Stage I lung adenocarcinoma lesions already harbor significantly altered $\mathrm{T}$ cells and NK cell compartments. ${ }^{35}$ The authors examined whether the immune composition of the tumor differed between earlystage (stage I) and later-stage (stages II and III) tumors. They determined that almost all immune changes detected in laterstage tumor lesions were already present in stage I tumors. The distribution of immune cell subset's frequency did not significantly differ across the tumor stages. Interestingly, NK cells were strongly reduced in tumors, and the few remaining NK cells expressed lower levels of granzyme B and IFN- $\gamma$ compared to the normal lung NK cells. ${ }^{35}$

Cancer can be initiated by genetic events that activate proto-oncogenes and inactivate tumor-suppressor genes; however, there is limited research on oncogenes and oncosuppressors associated with the immune system. Recent evidence indicates that oncogenes may directly regulate immune responses leading, in part, to immunosuppression. An innate immunosurveillance mechanism is causally linked to cancer-specific genetic lesions. NKG2D is an activating receptor expressed on human and mouse NK cells that recognizes a diverse family of ligands. Engagement of NKG2D activates the cytotoxic activity of NK cells against cells expressing these ligands. Therefore, tumor cells expressing NKG2D ligands could be eliminated by NKG2D+ cells, including NK cells. Because oncogenes drive malignant cells to apoptosis resistance and uncontrolled proliferation by altered expression of many critical genes, the expression of NKG2D ligands may also be affected by oncogenes. In fact, the $c-M y c$ oncogene can upregulate the expression of NK cell-activating ligands in cancer cells, making them targets of NK cell cytotoxicity: there is evidence that NKG2D ligands are induced on spontaneously arising tumors in a murine model of lymphomagenesis (surface expression of Rae1) and that $c-M y c$ is involved in their regulation. ${ }^{36}$ Transcription of NKG2D ligands is differentially regulated by the k-ras, c-Myc and Wnt pathways. ${ }^{37}$ Inhibition of $k$-ras and $c-M y c$ increased the expression of NKG2D ligands (such as MICA, MICB, ULBP1 and ULBP2) and enhanced the susceptibility of cancer cells to NK cells. The authors proposed that the cytotoxicity of NK cells exclusively depended on the amount of surface NKG2D ligands. Another work found that the deletion of the tumor-suppressor $\mathrm{Rb}$, overexpression of a mutated proto-oncogene Ras or both in cancer cells can alter the expression of NK cell receptor ligands, such as MHC-I,
Rae $1 \alpha$ and $\operatorname{Rae} 1 \delta$, that are associated with resistance to NK cell-mediated cytotoxicity. ${ }^{38}$ Ras activation in mouse and human cancer cells has been reported to upregulate the expression of NKG2D and Raet1 protein family members Rae $1 \alpha$ and $\operatorname{Rae} 1 \beta .{ }^{39}$

Oncogenic stress by Myc expression has been shown to induce DNA damage through a variety of mechanisms. In turn, DNA damage can also lead to NKG2D ligand expression. Mouse and human NKG2D ligands can be upregulated in nontumor cell lines by genotoxic stress and stalled DNA replication, conditions known to activate a major DNA damage checkpoint pathway initiated by ATM or ATR protein kinases. ${ }^{40}$ Thus, the DNA damage response may participate in activation of the immune system (particularly NK cells) against cancer cells.

Interestingly, Myc is known to regulate the expression of CD47 and CD274 (PD-L1 or B7-H1). Direct binding of Myc with the promoters of the CD47 and PD-L1 genes has been identified. ${ }^{41}$ Suppression of Myc in mouse and human tumor cells decreased the levels of CD47 and PD-L1 mRNA and protein, while $M y c$ inactivation attenuated CD47 and PD-L1 expression and enhanced the antitumor immune response. In contrast, when $M y c$ was inhibited in tumors with constitutive expression of CD47 or PD-L1, the immune response was inhibited and tumors continued to grow. ${ }^{41}$ Thus, $M y c$ expression may initiate and sustain tumorigenesis in part through the regulation of immune mechanisms. Furthermore, c-Myc is involved in IL-15 signaling pathway, which is critical for NK cell maturation and homeostasis. ${ }^{42}$ It has been reported that the overexpression of $c-M y c$ during NK cell development contributes to the overall transcription of multiple KIR genes. Binding of endogenous c-Myc to the distal promoter element is significantly enhanced upon IL-15 stimulation of peripheral blood NK cells and correlates with an increase in KIR transcription. This provides a direct mechanistic link between NK cell activation signals and KIR expression required for acquisition of the effectors' function during NK cell education. ${ }^{43}$

\section{Oncogenes in the regulation of immune cell metabolism}

c-Myc functions as a transcription factor promoting expression of numerous target genes to coordinate cell death, proliferation and metabolism. ${ }^{44}$ Beyond cancer, Myc is involved in many physiological and pathophysiological pathways, including the regulation of immunity and development of immune-mediated diseases, where Myc controls the expression of target genes to synchronize proliferation, death and 
metabolism at the cellular, tissue and organism levels. ${ }^{45}$ It has been reported that Myc family members play important roles in regulating the differentiation and activation of both innate and adaptive immune cells during development and after immune stimulation. c-Myc participates in the B cell receptor $(\mathrm{BCR})$-mediated transcriptional network, where it plays an important role in maintaining B cell homeostasis by regulating $\mathrm{B}$ cell growth, differentiation and apoptosis. c-Myc-negative B cells have a weak response to BCR signaling and are resistant to apoptosis. ${ }^{46}$ Furthermore, Myc expression results in sustained increases in intracellular $\mathrm{Ca}^{2+}$ and NFAT nuclear translocation, which are required for Myc to stimulate $\mathrm{B}$ cell proliferation and differentiation. ${ }^{47}$ These results revealed a novel mechanism by which Myc amplifies $\mathrm{Ca}^{2+}$ signals in $\mathrm{B}$ cells, thereby enabling the concurrent expression of Myc- and $\mathrm{Ca}^{2+}$-regulated target genes.

Metabolic reprogramming is a hallmark of cancer progression. ${ }^{48,49}$ Given that immune cells have emerged as central players in tumor growth and progression, metabolic reprogramming of immune cells in cancer and its contribution in cancer progression have been well investigated. ${ }^{50}$ However, the involvement of oncogenes in these alterations in immune cells is not well characterized. New evidence suggests that by controlling the expression of a wide array of metabolic genes in immune cells, Myc family members organize metabolic programs to sustain immune functions. ${ }^{51} \mathrm{c}$-Myc regulates the proliferation of $\mathrm{T}$ cells primarily through controlling the cell cycle. ${ }^{52}$ Myc also has an essential role in the upregulation of glutaminolysis upon activation of T cells, potentially through the mTOR pathway. ${ }^{53}$ These studies implicate Myc as an essential coordinator of $\mathrm{T}$ cell activation-induced cell growth, proliferation and metabolic reprogramming. Moreover, it is important to note that $\mathrm{T}$ cell activation-induced glycolysis and glutaminolysis are reminiscent of the metabolic changes in tumor cells, where both anaerobic glycolysis and glutaminolysis are induced by oncogenic signals.

p53 mutations are the most common cancer-associated mutations. The proneness of p53-knockout mice to develop $\mathrm{T}$ cell lymphomas underlines the role of TP53 in regulating $\mathrm{T}$ cell proliferation. ${ }^{54}$ In addition, the synergy between c-Myc and TP53 in establishing T cell malignancy has also been reported. ${ }^{55} p 53$ is induced in activated T cells and contributes to $\mathrm{T}$ cell homeostasis, ${ }^{56}$ thereby both $c-M y c$ and $p 53$ are induced upon $\mathrm{T}$ cell activation. Induction of TP53 leads to reduced expression of $c-M y c$, while inhibition of c-Myc results in lower levels of TP53. ${ }^{57}$ Thus, one can conclude that the c-Myc-p53 feedback mechanism contributes to the cessation of $\mathrm{T}$ cell proliferation in the immune response.
Induction of c-Myc following activation of T cells contributes to upregulation of TP53, which in turn can downregulate c-Myc levels. This will contribute to cessation of $\mathrm{T}$ cell proliferation and to $\mathrm{T}$ cell homeostasis. ${ }^{57}$

\section{Oncogenes and transcription factors as regulators of immune cell maturation}

The antitumor capacity of NK cells, as well as other immune effector cells, depends on their development, maturation and homeostasis. The assumption of immaturity of NK cells as a reason for their functional insufficiency in lung cancer and malignant melanoma was published for the first time by Sibbitt et al in $1984 .^{58}$ The results revealed a normal number of NK cells, which displayed reduced cytotoxic activity; this functional deficiency of NK cells was not due to the suppressive effect of other cells. Unusual disruption of the maturation of NK cells, independent of the negative influence of T cells, was also demonstrated in the mouse model. It was experimentally proved that the NK-ripening block was not mediated by $\mathrm{T}$ lymphocytes; no direct contact with tumor cells or microenvironment was required. ${ }^{59}$ Studies of patients with breast cancer showed a significant increase in the total number of activated peripheral blood NK cells. ${ }^{60}$ Similarly, another study of NK cells in peripheral blood of patients with breast cancer showed a significant increase in immature NK cells in the total cell pool. ${ }^{61}$ Heterogeneity and plasticity of NK cells was also apparent in these patients, which indicates an inhibition of NK cell differentiation in cancer. ${ }^{62}$

The hypothesis of an inhibition of the maturation and differentiation of NK cells is supported by the analysis of transcription factors responsible for these processes. The functional development of NK cells largely depends on transcription factors such as the Eomes. It was shown that maturation of NK cells, characterized by acquisition of a diverse repertoire of activating and inhibitory Ly49 family receptors, depends on Eomes. Deletion of Eomes from mature NK cells caused reversion to a more immature state. ${ }^{63}$ Loss of both T-bet and Eomes from mature NK cells resulted in loss of classical NK antigens. ${ }^{64}$ Thus, expression and function of T-bet and Eomes define the key genetically separable molecular checkpoints of NK cell maturation. ${ }^{65}$ The sensitivity to cancer, in particular to melanoma, was enhanced in the absence of T-bet due to the defects in maturation and development of NK cells in vivo. T-bet appears to be particularly important for NK cell control of metastatic disease in mouse models. ${ }^{66-68}$

The persistent hyperactivity of the transcription factor - STAT5 - in NK cells was revealed in patients with 
melanoma and metastatic renal cell carcinoma. ${ }^{69}$ STAT5 is responsible for maturation of NK cells. ${ }^{70}$ In addition, it is a proto-oncogene, and its hyperactivation accompanies the development of many malignant neoplasms. ${ }^{71}$ Analysis of gene expression in freshly isolated and ex vivo-cultured NK cells obtained from patients with progressive cancers also revealed PI3K/AKT hyperactivation. ${ }^{72}$ In our recent study, c-Myc expression in peripheral NK cells from patients with cancer was estimated by the Smart Flare method for the first time. c-Myc expression was significantly decreased in NK cells in all cancer patients independently of tumor location, stage of disease or the presence of metastases. ${ }^{73}$ Marked changes in the expression of other oncogenes, such as c-kit, STAT3 and soluble and membrane-bound forms of ligand for c-kit/stem cell factor (SCF), were also found in these NK cells. c-Kit expression in NK cells was significantly decreased in all patients. Expression of SCF had a clear tendency to be decreased, as well as c-kit did. ${ }^{73}$ Alterations of the c-kit/ SCF autocrine loop in NK cells in cancer seem indicated. In contrast, STAT3 expression in NK cells was significantly increased in lung cancer patients compared to healthy control donors. The disturbances of c-kit and c-Myc expression in NK cells may cause the reduced viability of NK cells, which may contribute to the decreased antitumor cytotoxicity of NK cells in patients with cancer. ${ }^{73}$

In addition to NK cell mechanisms, Myc plays an important role in cancer-related macrophages. c-Myc is induced in human macrophages during an alternative in vitro activation suggesting it plays a role in the polarization of macrophages. ${ }^{74}$ Macrophage-polarized activation is involved in immune responses and tumor biology. In addition, immunohistochemical analysis of colon and breast tumor samples has identified c-Myc expression in human tumor-associated macrophages (TAMs). c-Myc is expressed in vivo in human TAMs but not in resting macrophages. ${ }^{75}$ The authors note an interesting fact that c-Myc was induced in macrophages exposed in vitro to conditioned medium from different human tumor cell lines and was detected in CD68-positive macrophages-infiltrating human colon tumors. They hypothesized that there was a common mechanism of c-Myc upregulation in human TAMs in different tumor microenvironments. They concluded that c-Myc is a key element in alternative macrophage activation through the induction of alternative activation genes, likely via STAT6 and PPAR signaling. ${ }^{76} \mathrm{c}-\mathrm{Myc}$ also controls the expression of other important molecules (MMP9, VEGF, HIF-1 and TGF- $\beta$ ) in myeloid cell differentiation and polarization. ${ }^{77}$

Other oncogenes may also be involved in alternatively activated type 2 macrophage (M2) polarization. Endogenous p53 regulates M2 polarization in inflammation and during LPS-associated tolerance in vitro, ex vivo and in vivo. ${ }^{78,79}$ The authors suggest that p53 has an urgent role in regulating M2 polarization. Maintaining p53 in a state of low activity in M2-polarized cells is required to facilitate M2 gene expression. Thus, p53 may reduce the density and the protumoral phenotype of M2-like TAMs. Recent reports confirm such a role of p53 in TAM biology. ${ }^{80,81}$

\section{Mutations of oncogenes in immune cells in cancer}

There are several complimentary theories explaining the initiation of carcinogenesis, and the basic and widely accepted theory is the mutation theory, according to which cancer develops from a single tumor cell. Per this theory, cancer in the human body arises from the accumulation of mutations in DNA, which lead to the formation of defective proteins. The founder of the theory is the German biologist Teodor Boveri. In 1914, he suggested that disorders in chromosomes could lead to cancer. Later, these disorders were qualified by him as mutation-associated diseases. Mutations in oncogenes and suppressor genes are central to these theories, ${ }^{82}$ and mutations that occur in somatic cells can affect immune cells as well. In fact, there are serious abnormalities in the cytotoxic activity and in the number of NK cells in patients with a mutation in GATA2. The fact that it was the GATA2 mutation that caused these defects was confirmed by sequencing of DNA derived from a stored IL-2-dependent $\mathrm{T}$ cell line obtained from these patients. Although the NK cell population appeared to express low levels of GATA2, its expression was almost exclusively restricted to the $\mathrm{CD} 56^{\text {bright }}$ cell subset. ${ }^{83}$ Densitometry analysis showed a 15-fold increase in GATA2 expression in CD56 $6^{\text {bright }}$ NK cells compared with CD56 ${ }^{\mathrm{dim}}$ cells. The authors noted a decrease in the expression of CD117 and NKG2D in these patients. GATA2 regulates cell surface markers required for NK cell development, such as CD117. Therefore, GATA2 is a cell-intrinsic factor required for NK cell maturation and the generation or survival of CD56 $6^{\text {bright }}$ cells. GATA2 mutation is a likely cause of a classical NK cell deficiency.

Recently, DNA sequences of cells obtained from breast cancer patients have revealed mutations in the genes related specifically to immunity ${ }^{84}$ Although tumor viruses have been found in the normal breast, most women are infection free. It might be assumed that the development of cancer requires compromise of antiviral immunity. ${ }^{85}$ It was also reported that of the 1,175 shared genes that had mutations in the initial set of breast and cervical cancers, approximately 385 genes (33\%) could be involved in encoding proteins impacting the 
immune system. ${ }^{86}$ The author assessed 303 breast and cervical cancer samples, and all 303 specimens had mutations in multiple genes that could cause some deficiencies in immune responses. For example, Granzyme $\mathrm{A}$ and $\mathrm{H}$ genes were mutated in two breast cancers, and perforin 1 was mutated in another sample. In another report, mutations of kinases and the ubiquitin system in immune cells were investigated due to the relationship between phosphorylation and ubiquitylation events in the innate immune signaling networks. Almost $10 \%$ of all human genes encode proteins that control reversible phosphorylation or ubiquitylation of proteins. ${ }^{87}$ Moreover, mutations in other proteins, such as ligands, receptors and adaptors, affect protein phosphorylation and ubiquitylation indirectly by suppressing or enhancing the activation of particular signaling networks. ${ }^{87}$

Reevaluation of the role of the transcription factor c-Myc in the physiology of germinal center (GC) B cells ${ }^{88}$ demonstrated that lymphomas of human GC B cells with chromosomal translocation of Myc are observed in almost all sporadic lymphomas of Burkett and in diffuse large B lymphoma cells. ${ }^{89} \mathrm{~B}$ cell lymphomas, the most common type of human lymphoid malignancy in general, occur mainly from GC B cells. In the GC microenvironment, somatic hypermutation occurs, in which Myc transcription is required. c-Myc-positive GC B cells were identified in mice and humans. These cells are susceptible to translocation of the gene coding for c-Myc and can represent a stage of differentiation of cells with an especially high risk of malignant transformation. ${ }^{88}$

\section{Conclusion}

Evasion of immune defense by cancer is one of the key mechanisms of carcinogenesis. Studies in this area have been intensively conducted all over the world and accelerated development of novel and efficient immunotherapeutic approaches. The novel view of this field - the relationship between the expression of oncogenes and the defects in the immune system - has not yet been fully evaluated or comprehensively discussed. Understanding this new pathway of cancer-associated immune dysfunction has broad implications: it may suggest that the mechanisms of "oncogenetargeted drug therapy" may overlap with immunotherapy. For instance, Myc regulates the expression of two immune checkpoint proteins on the tumor cell surface - CD47 and PD-L1 - by directly binding to their gene promoters. The inactivation of Myc in mouse models of cancer led to the lower expression of CD47 and PD-L1, enhancing the antitumor immune response. ${ }^{41}$
Another example represents a different scenario where inhibition of receptor tyrosine kinase c-MET can improve cancer immunotherapy not via its effect on c-MET activity in malignant cells but by affecting the interaction of immunesuppressive neutrophils with tumor-specific CD8+ T lymphocytes. ${ }^{90}$ Inhibitors of the receptor tyrosine kinase c-MET are currently used in the clinic to target oncogenic signaling in cancerous cells. Using the murine tumor model, the authors reported that concomitant c-MET inhibition promoted adoptive $\mathrm{T}$ cell transfer and checkpoint immunotherapies by promoting tumor infiltration by effector T cells. Mechanistically, c-MET inhibition reduced the reactive mobilization and recruitment of neutrophils into the tumor site and draining lymph nodes in response to immunotherapy. In the absence of c-MET inhibition, neutrophils recruited to $\mathrm{T}$ cell-rich areas rapidly acquired immunosuppressive properties, preventing expansion of T lymphocytes and their effector functions. ${ }^{90}$

More studies are needed to determine the potentially negative impact of oncogene-targeting therapy in clinical settings and to design promising strategies to avoid disrupting the beneficial effects of oncogene on antitumor immune functions. This work could also have implications for significantly improving the efficacy of immune-based cancer therapies. In addition, understanding the role of oncogenes in immune cells for potentially predicting and controlling the benefits and disadvantages of oncogene-targeting therapy in antitumor immune functions, especially when combined with immunotherapeutic approaches, is a very important, but undeveloped area of clinical research. Future basic and clinical studies investigating targeted treatment and immunotherapies will reveal even more about how oncogenes use the immune system to support tumor development and progression, as well as how they can be used to cure cancer and metastatic disease.

\section{Disclosure}

The authors report no conflicts of interest in this work.

\section{References}

1. Thomas RK, Baker AC, RM Debiasi, et al. High-throughput oncogene mutation profiling in human cancer. Nat Genet. 2007;39(3):347-351.

2. Shurin M. Cancer as an immune-mediated disease. Immunotargets Ther. 2012;1:1-6.

3. Weinstein IB. Cancer. Addiction to oncogenes--the Achilles heal of cancer. Science. 2002;297(5578):63-64.

4. Weinstein IB, Joe A. Oncogene addiction. Cancer Res. 2008;68(9):30773080; discussion 3080 .

5. Weinstein B. Relevance of the concept of oncogene addiction to hormonal carcinogenesis and molecular targeting in cancer prevention and therapy. Adv Exp Med Biol. 2008;617:3-13.

6. Sharma SV, Settleman J. Oncogene addiction: setting the stage for molecularly targeted cancer therapy. Genes Dev. 2007;21(24):3214-3231. 
7. Bachireddy P, Rakhra K, Felsher DW. Immunology in the clinic review series; focus on cancer: multiple roles for the immune system in oncogene addiction. Clin Exp Immunol. 2012;167(2):188-194.

8. Restifo NP. Can antitumor immunity help to explain "oncogene addiction"? Cancer Cell. 2010;18(5):403-405.

9. Felsher DW. MYC inactivation elicits oncogene addiction through both tumor cell-intrinsic and host-dependent mechanisms. Genes Cancer. 2010;1(6):597-604.

10. Muller J, Samans B, van Riggelen J, et al. TGFbeta-dependent gene expression shows that senescence correlates with abortive differentiation along several lineages in Myc-induced lymphomas. Cell Cycle. 2010;9(23):4622-4626.

11. Savas P, Caramia F, Teo ZL, Loi S. Oncogene addiction and immunity: clinical implications of tumour infiltrating lymphocytes in breast cancers overexpressing the HER2/neu oncogene. Curr Opin Oncol. 2014;26(6):562-567.

12. Sibbitt WL Jr. Oncogenes, growth factors, and autoimmune diseases (review). Anticancer Res. 1991;11(1):97-113.

13. Maas K, Chan S, Parker J, et al. Cutting edge: molecular portrait of human autoimmune disease. J Immunol. 2002;169(1):5-9.

14. Aune TM, Maas K, Parker J, Moore JH, Olsen NJ. Profiles of gene expression in human autoimmune disease. Cell Biochem Biophys. 2004;40(2):81-96.

15. Salman T. Spontaneous tumor regression. J Oncol Sci. 2016;2:1-4.

16. Abdelrazeq AS. Spontaneous regression of colorectal cancer: a review of cases from 1900 to 2005. Int J Colorectal Dis. 2007;22(7): 727-736.

17. Printz C. Spontaneous regression of melanoma may offer insight into cancer immunology. J Natl Cancer Inst. 2001;93(14):1047-1048.

18. Halliday GM, Patel A, Hunt MJ, Tefany FJ, Barnetson RS. Spontaneous regression of human melanoma/nonmelanoma skin cancer: association with infiltrating CD4+ T cells. World J Surg. 1995;19(3):352-358.

19. Stoll BA. Spontaneous regression of cancer: new insights. Biotherapy. 1992;4(1):23-30.

20. Jessy T. Immunity over inability: the spontaneous regression of cancer. J Nat Sci Biol Med. 2011;2(1):43-49.

21. Thomas JA, Badini M. The role of innate immunity in spontaneous regression of cancer. Indian J Cancer. 2011;48(2):246-251.

22. Choi N, Cho JK, Baek CH, Ko YH, Jeong HS. Spontaneous regression of metastatic cancer cells in the lymph node: a case report. BMC Res Notes. 2014;7:293.

23. Ovestad IT, Gudlaugsson E, Skaland I, et al. Local immune response in the microenvironment of CIN2-3 with and without spontaneous regression. Mod Pathol. 2010;23(9):1231-1240.

24. Croxford JL, Tang ML, Pan MF, et al. ATM-dependent spontaneous regression of early Emu-myc-induced murine B-cell leukemia depends on natural killer and T cells. Blood. 2013;121(13):2512-2521.

25. Raulet DH, Guerra N. Oncogenic stress sensed by the immune system: role of natural killer cell receptors. Nat Rev Immunol. 2009;9(8):568-580.

26. Rocca YS, Roberti MP, Arriaga JM, et al. Altered phenotype in peripheral blood and tumor-associated NK cells from colorectal cancer patients. Innate Immun. 2013;19(1):76-85.

27. Levy EM, Roberti MP, Mordoh J. Natural killer cells in human cancer: from biological functions to clinical applications. J Biomed Biotechnol. 2011;2011:676198.

28. Messaoudene M, Frazao A, Gavlovsky PJ, Toubert A, Dulphy N, Caignard A. Patient's natural killer cells in the era of targeted therapies: role for tumor killers. Front Immunol. 2017;8:683.

29. Platonova S, Cherfils-Vicini J, Damotte D, et al. Profound coordinated alterations of intratumoral NK cell phenotype and function in lung carcinoma. Cancer Res. 2011;71(16):5412-5422.

30. Mamessier E, Sylvain A, Bertucci F, et al. Human breast tumor cells induce self-tolerance mechanisms to avoid NKG2D-mediated and DNAM-mediated NK cell recognition. Cancer Res. 2011;71(21): $6621-6632$.
31. Mamessier E, Sylvain A, Thibult ML, et al. Human breast cancer cells enhance self tolerance by promoting evasion from NK cell antitumor immunity. J Clin Invest. 2011;121(9):3609-3622.

32. Messaoudene M, Fregni G, Fourmentraux-Neves E, et al. Mature cytotoxic CD56(bright)/CD16(+) natural killer cells can infiltrate lymph nodes adjacent to metastatic melanoma. Cancer Res. 2014;74(1):81-92.

33. Fregni G, Messaoudene M, Fourmentraux-Neves E, et al. Phenotypic and functional characteristics of blood natural killer cells from melanoma patients at different clinical stages. PLoS One. 2013;8(10):e76928.

34. Garcia-Iglesias T, Del Toro-Arreola A, Albarran-Somoza B, et al. Low NKp30, NKp46 and NKG2D expression and reduced cytotoxic activity on NK cells in cervical cancer and precursor lesions. BMC Cancer. 2009;9:186.

35. Lavin Y, Kobayashi S, Leader A, et al. Innate immune landscape in early lung adenocarcinoma by paired single-cell analyses. Cell. 2017;169(4):750.e17-765.e17.

36. Unni AM, Bondar T, Medzhitov R. Intrinsic sensor of oncogenic transformation induces a signal for innate immunosurveillance. Proc Natl Acad Sci U S A. 2008;105(5):1686-1691.

37. Heo W, Lee YS, Bae J. Inhibition of oncogenes affects the expression of NKG2D ligands in cancer cells. J Life Sci. 2013;23(10):1216-1222.

38. Orozco-Morales M, Sanchez-Garcia FJ, Golan-Cancela I, et al. RB mutation and RAS overexpression induce resistance to NK cellmediated cytotoxicity in glioma cells. Cancer Cell Int. 2015;15:57.

39. Liu XV, Ho SS, Tan JJ, Kamran N, Gasser S. Ras activation induces expression of Raet1 family NK receptor ligands. J Immunol. 2012;189(4):1826-1834.

40. Gasser S, Orsulic S, Brown EJ, Raulet DH. The DNA damage pathway regulates innate immune system ligands of the NKG2D receptor. Nature. 2005;436(7054):1186-1190.

41. Casey SC, Tong L, Li Y, et al. MYC regulates the antitumor immune response through CD47 and PD-L1. Science. 2016;352(6282):227-231.

42. Bianchi T, Gasser S, Trumpp A, MacDonald HR. c-Myc acts downstream of IL-15 in the regulation of memory CD8 T-cell homeostasis. Blood. 2006;107(10):3992-3999.

43. Cichocki F, Hanson RJ, Lenvik T, et al. The transcription factor c-Myc enhances KIR gene transcription through direct binding to an upstream distal promoter element. Blood. 2009;113(14):3245-3253.

44. Nie Z, Hu G, Wei G, et al. c-Myc is a universal amplifier of expressed genes in lymphocytes and embryonic stem cells. Cell. 2012;151(1): 68-79.

45. Gnanaprakasam JNR, Sherman JW, Wang R. MYC and HIF in shaping immune response and immune metabolism. Cytokine Growth Factor Rev. 2017;35:63-70.

46. Murn J, Mlinaric-Rascan I, Vaigot P, Alibert O, Frouin V, Gidrol X. A Myc-regulated transcriptional network controls B-cell fate in response to BCR triggering. BMC Genomics. 2009;10:323.

47. Habib T, Park H, Tsang M, et al. Myc stimulates B lymphocyte differentiation and amplifies calcium signaling. J Cell Biol. 2007;179(4) $717-731$.

48. Hanahan D, Weinberg RA. Hallmarks of cancer: the next generation. Cell. 2011;144(5):646-674.

49. Ward PS, Thompson CB. Metabolic reprogramming: a cancer hallmark even Warburg did not anticipate. Cancer Cell. 2012;21(3):297-308

50. Biswas SK. Metabolic reprogramming of immune cells in cancer progression. Immunity. 2015;43(3):435-449.

51. Gnanaprakasam JN, Wang R. MYC in regulating immunity: metabolism and beyond. Genes (Basel). 2017;8(3):88.

52. Wang R, Dillon CP, Shi LZ, et al. The transcription factor Myc controls metabolic reprogramming upon T lymphocyte activation. Immunity. 2011;35(6):871-882.

53. Dose M, Khan I, Guo Z, et al. c-Myc mediates pre-TCR-induced proliferation but not developmental progression. Blood. 2006;108(8):2669-2677.

54. Donehower LA, Harvey M, Vogel H, et al. Effects of genetic background on tumorigenesis in p53-deficient mice. Mol Carcinog. 1995;14(1):16-22. 
55. Blyth K, Terry A, O'Hara M, et al. Synergy between a human c-myc transgene and p53 null genotype in murine thymic lymphomas: contrasting effects of homozygous and heterozygous p53 loss. Oncogene. 1995;10(9):1717-1723.

56. Madapura HS, Salamon D, Wiman KG, et al. p53 contributes to T cell homeostasis through the induction of pro-apoptotic SAP. Cell Cycle. 2012;11(24):4563-4569.

57. Madapura HS, Salamon D, Wiman KG, Lain S, Klein E, Nagy N. cMycp53 feedback mechanism regulates the dynamics of $\mathrm{T}$ lymphocytes in the immune response. Cell Cycle. 2016;15(9):1267-1275.

58. Sibbitt WL Jr, Bankhurst AD, Jumonville AJ, Saiki JH, Saiers JH, Doberneck RC. Defects in natural killer cell activity and interferon response in human lung carcinoma and malignant melanoma. Cancer Res. 1984;44(2):852-856.

59. Richards JO, Chang X, Blaser BW, Caligiuri MA, Zheng P, Liu Y. Tumor growth impedes natural-killer-cell maturation in the bone marrow. Blood. 2006;108(1):246-252.

60. Farazmand S, Amani D, Hassan Z-M. Investigation of NK cell population in peripheral blood and tumor lesions of patients with breast cancer. Iranian J Immunol. 2005;2(3):152-157.

61. Mamessier E, Pradel LC, Thibult ML, et al. Peripheral blood NK cells from breast cancer patients are tumor-induced composite subsets. J Immunol. 2013;190(5):2424-2436.

62. Mamessier E, Bourgin C, Olive D. When breast cancer cells start to fend the educational process of NK cells off. Oncoimmunology. 2013;2(12):e26688.

63. Townsend MJ, Weinmann AS, Matsuda JL, et al. T-bet regulates the terminal maturation and homeostasis of NK and Valpha14i NKT cells. Immunity. 2004;20(4):477-494.

64. Robbins SH, Tessmer MS, Van Kaer L, Brossay L. Direct effects of T-bet and MHC class I expression, but not STAT1, on peripheral NK cell maturation. Eur J Immunol. 2005;35(3):757-765.

65. Gordon SM, Chaix J, Rupp LJ, et al. The transcription factors T-bet and Eomes control key checkpoints of natural killer cell maturation. Immunity. 2012;36(1):55-67.

66. Werneck MB, Lugo-Villarino G, Hwang ES, Cantor H, Glimcher LH. T-bet plays a key role in NK-mediated control of melanoma metastatic disease. J Immunol. 2008;180(12):8004-8010.

67. Gill S, Vasey AE, De Souza A, et al. Rapid development of exhaustion and down-regulation of eomesodermin limit the antitumor activity of adoptively transferred murine natural killer cells. Blood. 2012;119(24):5758-5768.

68. Peng SL, Townsend MJ, Hecht JL, White IA, Glimcher LH. T-bet regulates metastasis rate in a murine model of primary prostate cancer. Cancer Res. 2004;64(2):452-455.

69. Varker KA, Kondadasula SV, Go MR, et al. Multiparametric flow cytometric analysis of signal transducer and activator of transcription 5 phosphorylation in immune cell subsets in vitro and following interleukin-2 immunotherapy. Clin Cancer Res. 2006;12(19):5850-5858.

70. Eckelhart E, Warsch W, Zebedin E, et al. A novel Ncr1-Cre mouse reveals the essential role of STAT5 for NK-cell survival and development. Blood. 2011;117(5):1565-1573.
71. Koppikar P, Lui VW, Man D, et al. Constitutive activation of signal transducer and activator of transcription 5 contributes to tumor growth, epithelial-mesenchymal transition, and resistance to epidermal growth factor receptor targeting. Clin Cancer Res. 2008;14(23):7682-7690.

72. Park KU, Jin P, Sabatino M, et al. Gene expression analysis of ex vivo expanded and freshly isolated NK cells from cancer patients. J Immunother. 2010;33(9):945-955.

73. Zakiryanova GK, Kustova E, Urazalieva NT, et al. Alterations of oncogenes expression in NK cells in patients with cancer. Immun Inflamm Dis. 2017;5(4):493-502.

74. Pello OM, De Pizzol M, Mirolo M, et al. Role of c-MYC in alternative activation of human macrophages and tumor-associated macrophage biology. Blood. 2012;119(2):411-421.

75. Pello OM, Chevre R, Laoui D, et al. In vivo inhibition of c-MYC in myeloid cells impairs tumor-associated macrophage maturation and pro-tumoral activities. PLoS One. 2012;7(9):e45399.

76. Pello OM. Macrophages and c-Myc cross paths. Oncoimmunology. 2016;5(6):e1151991.

77. Li L, Ng DS, Mah WC, et al. A unique role for $\mathrm{p} 53$ in the regulation of M2 macrophage polarization. Cell Death Differ. 2015;22(7):1081-1093.

78. Lowe JM, Menendez D, Bushel PR, et al. p53 and NF-kappaB coregulate proinflammatory gene responses in human macrophages. Cancer Res. 2014;74(8):2182-2192.

79. Lowe JM, Menendez D, Fessler MB. A new inflammatory role for p53 in human macrophages. Cell Cycle. 2014;13(19):2983-2984.

80. Lujambio A, Akkari L, Simon J, et al. Non-cell-autonomous tumor suppression by p53. Cell. 2013;153(2):449-460.

81. Vineis P, Schatzkin A, Potter JD. Models of carcinogenesis: an overview. Carcinogenesis. 2010;31(10):1703-1709.

82. Sonnenschein C, Soto AM. Theories of carcinogenesis: an emerging perspective. Semin Cancer Biol. 2008;18(5):372-377.

83. Mace EM, Hsu AP, Monaco-Shawver L, et al. Mutations in GATA2 cause human NK cell deficiency with specific loss of the CD56(bright) subset. Blood. 2013;121(14):2669-2677.

84. Friedenson B. Mutations in components of antiviral or microbial defense as a basis for breast cancer. Funct Integr Genomics. 2013;13(4): 411-424.

85. Friedenson B. Many breast cancer mutations parallel mutations in known viral cancers. J Genome Exomes. 2014;3:17-35.

86. Friedenson B. Mutations in breast cancer exome sequences predict susceptibility to infections and converge on the same signaling pathways. J Genome Exomes. 2015;4:1-28.

87. Cohen P. Immune diseases caused by mutations in kinases and components of the ubiquitin system. Nat Immunol. 2014;15(6):521-529.

88. Calado DP, Sasaki Y, Godinho SA, et al. The cell-cycle regulator c-Myc is essential for the formation and maintenance of germinal centers. Nat Immunol. 2012;13(11):1092-1100.

89. Klein U, Dalla-Favera R. Germinal centres: role in B-cell physiology and malignancy. Nat Rev Immunol. 2008;8(1):22-33.

90. Glodde N, Bald T, van den Boorn-Konijnenberg D, et al. Reactive neutrophil responses dependent on the receptor tyrosine kinase c-MET limit cancer immunotherapy. Immunity. 2017;47(4):789.e9-802.e9.
ImmunoTargets and Therapy

\section{Publish your work in this journal}

ImmunoTargets and Therapy is an international, peer-reviewed open access journal focusing on the immunological basis of diseases, potential targets for immune based therapy and treatment protocols employed to improve patient management. Basic immunology and physiology of the immune system in health, and disease will be also covered. In addition, the journal will focus on the impact of manage-

\section{Dovepress}

ment programs and new therapeutic agents and protocols on patient perspectives such as quality of life, adherence and satisfaction. The manuscript management system is completely online and includes a very quick and fair peer-review system, which is all easy to use. Visit http://www.dovepress.com/testimonials.php to read real quotes from published authors. 